This work is supported in part by a grant of Wisconsin Alumni Research Foundation funds made by the Graduate Research Committee.

1. P. C. HAMMER \& A. W. WyMORE, "Numerical integration over higher dimensional regions." Unpublished manuscript. 1939.

2. G. SzEGÖ, Orthogonal Polynomials, Am. Math. Soc. Colloguium Publications, v. 23, New York,

3. G. W. TYLER, "Numerical integration of functions of several variables," Canadian J. Math., v. 5,1953 , p. $393-412$.

\title{
Numerical Integration over Simplexes
}

1. Introduction. Integration formulae for numerical evaluation of integrals over the simplex in $n$-space have been given inductively by Hammer, Marlowe, and Stroud [1] so that it is possible in principle to determine a formula holding exactly for the $k$ th degree polynomial in $n$ variables. In the same paper certain affinely symmetric integration formulae are given for the triangle and tetrahedron. Using the theory proposed by Hammer and Wymore [2], it is possible to extend the usefulness of methods developed by transformations of the regions and by use of Cartesian products.

In this paper we give two integration formulae of affinely symmetric type for the simplex in $n$-space which respectively hold exactly for the quadratic polynomial and the cubic polynomial in $n$ variables. The method for establishing the exact values of integrals needed we believe is new in that the "numerical" formulae are used for the purpose.

2. The formula for cubic polynomials. Let the vertices of the $n$-simplex, $S_{n}$, be $V_{0}, \cdots, V_{n}$ and then its centroid is given by $C=\sum_{1}^{n} V_{i} /(n+1)$. Let $\Delta_{n}$ be the hypervolume of $S_{n}$.

THEOREM 1: An integration formula exact for the general cubic polynomial over $S_{n}$ for $n \geq 1$ is given by

$$
\int_{S_{n}} f d v_{n}=a_{n} \sum_{0}^{n} f\left(U_{i}\right)+c_{n} f(C)
$$

where

and

$$
a_{n}=\frac{(n+3)^{2}}{4(n+1)(n+2)} \Delta_{n} \quad c_{n}=\frac{-(n+1)^{2}}{4(n+2)} \Delta_{n}
$$

$$
U_{i}=\frac{2}{n+3} V_{i}+\frac{n+1}{n+3} C \quad i=0, \cdots, n .
$$

Proof: It may first be remarked that the points $U_{i}$ are on the median lines of $S_{n}$ and that the statement of the theorem is in symmetric form. In particular, under an affine transformation taking $S_{n}$ onto itself, the set of points $\left\{U_{i}\right\}$ is invariant and the centroid $C$ is fixed. Since there exists an affine transformation mapping any simplex in $E_{n}$ onto any other we may choose any particular simplex $S_{n}$ to carry out the proof. Our choice is specified by vertices as follows:

$$
\begin{array}{ll}
V_{0}=(0, \cdots, 0), & V_{1}=(1,0, \cdots, 0), \\
V_{2}=(1,1,0, \cdots, 0), \cdots, & V_{n}=(1,0, \cdots, 0,1) .
\end{array}
$$


It is simply verified that the formula given holds for $n=1$ and $n=2$. Hence we assume that it holds for $E_{n-1}$ and proceed to show that it also holds for $E_{n}$ where $n-1 \geq 2$. Let $f$ be a cubic polynomial in $x_{1}, x_{2}, \cdots, x_{n}$. Then $\left.f\right|_{x_{1}=1}$ is a cubic polynomial in $x_{2}, \cdots, x_{n}$. Now using a result established in [1] we may write

$$
\begin{aligned}
\int_{S_{n}} f d v_{n}=\int_{0}^{1}\left(\int_{x_{1} S_{n-1}} f d v_{n-1}\right) & d x_{1} \\
& =\int_{0}^{1} x_{1}^{n-1}\left[a_{n-1} \sum_{1}^{n} f\left(x_{1} \bar{U}_{i}\right)+c_{n-1} f\left(x_{1} \bar{C}_{i}\right)\right] d x_{1}
\end{aligned}
$$

where $S_{n-1}$ is the $(n-1)$-simplex with vertices $V_{1}, \cdots, V_{n}$ in the hyperplane $x_{1}=1, \bar{C}=1 / n \sum_{1}^{n} V_{i}, \bar{U}_{i}=\frac{2}{n+2} V_{i}+\frac{n}{n+2} \bar{C}, i=1,2, \cdots, n$ and $a_{n-1}$ and $c_{n-1}$ are the weights as indicated in the theorem with $n$ replaced by $n-1$. It is observed that the hypervolume $\Delta_{n-1}$ is $1 /(n-1)$ ! and $\Delta_{n}$ is $1 / n$ !. Let $f$ be the monomial $x_{1}{ }^{i} x_{2}{ }^{j} x_{3}{ }^{k}$ where $0 \leq i+j+k \leq 3$. Using (2) we find on substitution and simplification that:

$$
\int_{S_{n}} x_{1}{ }^{i} x_{2}{ }^{j} x_{3}{ }^{k} d v_{n}=\frac{\Delta_{n}\left[(n+2)^{2-j-k}\left(3^{j}+3^{k}+n-2\right)-n^{3-j-k}\right]}{4(n+1)(n+i+j+k)} .
$$

On the basis of our assumption, (3) gives the value of the integral indicated. On the other hand, formula (1) applied to $f=x_{1}{ }^{i} x_{2}{ }^{i} x_{3}{ }^{k}$ gives

$$
\frac{\Delta_{n}}{4(n+1)(n+2)}\left\{(n+3)^{2-i-j-k}\left[n^{i}+(n+2)^{i}\left(3^{j}+3^{k}+n-2\right)\right]-(n+1)^{3-i-j-k_{n} i}\right\} .
$$

Now it may then be directly verified that (4) gives the same result as the right of (3) for $0 \leq i+j+k \leq 3,0 \leq i \leq 3$ and $j \geq k$. Hence in view of the symmetry with which the last $n-1$ coordinates appear in the set of vertices $V_{0}, \cdots, V_{n},(4)$ is verified as correct for all monomials of form $x_{1}{ }_{1} x^{j}{ }_{i_{1}} x^{k}{ }_{i_{2}}$ for $0 \leq i+j+k \leq 3, i_{1} \neq i_{2}$ where $i_{1}$ and $i_{2}$ are taken from $2, \cdots, n$. The only monomial type thus omitted is $x_{2} x_{3} x_{4}$ provided $n \geq 4$. Using formula (2) for this monomial, we find

$$
\int_{S_{n}} x_{2} x_{3} x_{4} d v_{n}=\frac{\Delta_{n}}{4(n+1)(n+2)(n+3)}
$$

which coincides with the value obtained on substituting $f=x_{2} x_{3} x_{4}$ in (1). Hence the formula (1) holds for all required monomials and for the cubic polynomials provided it holds on $S_{n-1}$. Hence by complete induction the formula (1) holds.

3. A formula for the quadratic polynomial.

THEOREM 2: The formula

$$
\int_{S_{n}} f d v_{n}=\frac{\Delta_{n}}{n+1} \sum_{0}^{n} f\left(U_{i}\right)
$$


holds for the quadratic polynomial over $S_{n}, n \geq 1$ provided $U_{i}=r V_{i}+(1-r) C$ where either $r=1 / \sqrt{n+2}$ or $r=-1 / \sqrt{n+2}$. The choice of the positive sign for $r$ gives points $U_{i}$ inside $S_{n}$ for all $n$. If the negative sign is chosen, the points $U_{i}$ are outside $S_{n}$ for $n>2$.

The proof of this theorem may be made along the same lines as that of theorem 1 , by induction.

4. Remarks. The formulae given are affinely symmetrical. Those for the cubic polynomial are based on rational combinations of the vertices whereas the formulae for the quadratic function are based on irrational combinations unless $n+2$ is a perfect square. The weight of the centroid is negative and increases numerically with $n$ for the cubic case. The formula for the cubic polynomial may well be used as an exact integration formula for any polynomial of degree at most three. Since the general cubic polynomial has $(n+1)(n+2)(n+3) / 6$ terms, the formula (1) is hyperefficient (see [1] or [2]) for $n>3$ since it is based on $n+2$ evaluation points.

The practicality of using simplicial decompositions of regions decreases rapidly with increasing $n$. For $n=1$, the formula (1) is based on three evaluation points whereas Gauss' formula uses only two. We conjecture that for no region of bounded volume in $E_{n}$ for $n \geq 2$ will it be possible to obtain a numerical integration formula exact for the cubic polynomial based on fewer than $n+2$ points. Over the hypercube, Tyler [3] has shown that the cubic polynomial may be integrated exactly by a formula using $2 n$ points. Hammer and Wymore have extended this result to certain symmetrical regions.

Extension of affinely symmetric formulae for integration over the simplex to higher degree polynomials promises to offer significantly greater complexity. In the tetrahedron, for example, we have shown that evaluation points to obtain a formula exact for the fourth degree polynomial cannot all be taken on the median lines. However, the methods used here may be of use for higher degree polynomials.

University of Wisconsin

Preston C. Hammer

Madison, Wisconsin

Chemstrand Corp.

Arthur H. Stroud

Alabama

This research was supported by a grant of Wisconsin Alumni Research Foundation funds made by the Graduate Research Committee of the University of Wisconsin.

1. P. C. Hammer, O. J. Marlowe, \& A. H. Stroud, "Numerical integration over simplexes and cones" [see p. 130 this issue].

2. P. C. Hammer \& A. W. Wymore, "Numerical evaluation of multiple integrals." Unpublished manuscript.

3. G. W. TYLER, "Numerical integration of functions of several variables," Canadian Jn. Math., v. 5, 1953, p. 393-412. 